\title{
Motor self-regulation in goats (Capra aegagrus hircus) in a detour-reaching task
}

\author{
Jan Langbein Corresp. 1 \\ ${ }^{1}$ Institute of Behavioural Physiology, Leibniz Institute for Farm Animal Biology, Dummerstorf, Germany \\ Corresponding Author: Jan Langbein \\ Email address: langbein@fbn-dummerstorf.de
}

Motor self-regulation is the ability to inhibit a prepotent response to a salient cue in favour of a more appropriate response. Motor self-regulation is an important component of the processes that interact to generate effective inhibitory control of behaviour, and is theorized to be a prerequisite of complex cognitive abilities in humans and other animals. In a large comparative study using the cylinder task, motor self-regulation was studied in 36 different species, mostly birds and primates. To broaden the range of species to comprehensively evaluate this phenomenon, motor self-regulation was studied in the domestic goat, which is a social ungulate species and moderate food specialist. Using the cylinder task, goats were first trained to perform a detour-reaching response to retrieve a reward from an opaque cylinder. Subsequently, an otherwise identical transparent cylinder was substituted for the opaque cylinder over 10 test trials. The goats' ability to resist approaching the visible reward directly by touching the cylinder and to retain the trained detour-reaching response was measured. The results indicated that goats showed motor self-regulation at a level comparable to or better than that of many of the bird and mammal species tested to date. However, the individual reaction patterns revealed large intra- and inter-individual variability regarding motor self-regulation. An improvement across trials was observed only in latency to make contact with the reward; no improvement in the proportion of accurate trials was observed. A short, distinct pointing gesture by the experimenter during baiting did not have any impact on the side of the cylinder to which the goats detoured. In half of goats, individual side biases were observed when detouring to the side of the cylinder, but there was no bias at the population level for either the left or right side. The results underline the need for a detailed examination of individual performance and additional measures to achieve a complete understanding of animal performance in motor self-regulation tasks. 
2

3

4 Motor self-regulation in goats (Capra aegagrus hircus) in a detour-reaching

5 task

6

7

8 Jan Langbein

9

10

11

12 Leibniz Institute for Farm Animal Biology, Institute of Behavioural Physiology, Dummerstorf,

13 Germany

14

15

16

17 E-mail: langbein@fbn-dummerstorf.de

18

19 
21 Motor self-regulation is the ability to inhibit a prepotent response to a salient cue in favour of a

22 more appropriate response. Motor self-regulation is an important component of the processes

23 that interact to generate effective inhibitory control of behaviour, and is theorized to be a 24 prerequisite of complex cognitive abilities in humans and other animals. In a large comparative 25 study using the cylinder task, motor self-regulation was studied in 36 different species, mostly 26 birds and primates. To broaden the range of species to comprehensively evaluate this

27 phenomenon, motor self-regulation was studied in the domestic goat, which is a social ungulate 28 species and moderate food specialist. Using the cylinder task, goats were first trained to perform 29 a detour-reaching response to retrieve a reward from an opaque cylinder. Subsequently, an otherwise identical transparent cylinder was substituted for the opaque cylinder over 10 test trials. The goats' ability to resist approaching the visible reward directly by touching the cylinder and to retain the trained detour-reaching response was measured. The results indicated that goats showed motor self-regulation at a level comparable to or better than that of many of the bird and mammal species tested to date. However, the individual reaction patterns revealed large intraand inter-individual variability regarding motor self-regulation. An improvement across trials was observed only in latency to make contact with the reward; no improvement in the proportion of accurate trials was observed. A short, distinct pointing gesture by the experimenter during baiting did not have any impact on the side of the cylinder to which the goats detoured. In half of goats, individual side biases were observed when detouring to the side of the cylinder, but there was no bias at the population level for either the left or right side. The results underline the need for a detailed examination of individual performance and additional measures to achieve a 42 complete understanding of animal performance in motor self-regulation tasks. 


\section{Introduction}

In recent years, a large body of research has studied a behavioural phenomenon described as inhibitory control or self-control in various species of birds and mammals. This phenomenon is defined as an individual's ability to inhibit an impulsive or prepotent response, normally in reaction to a salient cue or stimulus, in favour of a more appropriate response (Bray et al. 2014; MacLean et al. 2014; Jelbert et al. 2016; Vernouillet et al. 2016). This type of behavioural control has been discussed as one of the prerequisites for problem solving and is assumed to be an important aspect of complex cognitive capabilities, such as reasoning and planning (Kralik et al. 2002; Diamond 2013), and regarded as essential for effectively interacting with the environment (Burke et al. 1991). Studies of different primate species have attributed welldeveloped inhibitory skills to living in complex social groups and have found that higher levels of fission-fusion dynamics are correlated with better inhibitory control and consequently higher behavioural flexibility (Amici et al. 2008; Maclean et al. 2013). Inhibitory control is a core component of the so-called executive functions (Miyake et al. 2000). The executive functions (Diamond 2013) comprise a cluster of top-down mental processes activated when a behaviour switches from automatic, instinctual or learned execution

61 to high-level control (insight, inference or reasoning) associated with greater cognitive effort. According to Diamond (2013), inhibitory control is based on several subdomains: cognitive and 63 attentional inhibition, which are subsumed under the category interference control, as well as 64 response inhibition. Diamond (2013) equates the latter with behavioural inhibition or self65 control. By contrast, Beran (2015) hierarchically separated response inhibition from self-control. 
According to his definition, response inhibition requires only the inhibition of a prepotent motor response, whereas self-control requires decision making as well. Other authors have classified response inhibition and self-control as separate processes under the generic term 'behavioural inhibition' (Bari \& Robbins 2013).

The most frequently used paradigms to compare inhibitory control across different mammal and bird species are the A-not-B task (Osthaus et al. 2012; Nawroth et al. 2015a), reversal-learning tasks (Tapp et al. 2003; Bond et al. 2007), delay-of-gratification tasks (Anderson et al. 2010; Hillemann et al. 2014) and detour-reaching tasks (Kabadayi et al., 2018). For the latter, either a transparent barrier (Pongracz et al. 2001; Vlamings et al. 2010; Baragli et al. 2011) or a transparent cylinder (Bray et al. 2014; MacLean et al. 2014) is often used which the animal must detour to reach a reward. In light of the debate regarding subdomains of inhibitory control (Diamond 2013) as well as the debate about the classification of response inhibition and self-control, it is questionable whether the different tasks mentioned above all measure the same aspects of inhibitory control (Manrique \& Call 2015; Brucks et al. 2017). The A-not-B task is strongly dependent on selective attention as it is affected by such contextual features as the number of boxes presented, the speed of movements and visual distinctiveness (Kabadayi et al. 2016). Reversal-learning tasks and especially delay-of-gratification tasks have been widely accepted as accurately measuring self-control (Evans et al. 2014; Beran 2015); however, performance may also be impacted by task-specific demands, which depend on other executive functions, such as working memory and cognitive flexibility (Manrique \& Call 2015).

This basic aspect of behavioural inhibition has recently been referred to as motor selfregulation (Bray et al. 2014; Kabadayi et al. 2016), and this term is used hereafter in this paper. It has been argued that detour-reaching tasks, such as the cylinder task, likely reflect 
89 fundamental inhibitory skills, which require only the inhibition of a prepotent response elicited 90 by a salient cue in favour of a more appropriate motor pattern (Bari \& Robbins 2013; Kabadayi 91 et al. 2018). In the cylinder task, subjects are first trained to locate a reward hidden inside a 92 horizontally oriented, opaque cylinder that is open at both sides. To retrieve the reward, subjects 93 must detour to the side of the cylinder. Once the animals are well trained in this task, an 94 otherwise identical transparent cylinder is substituted for the opaque cylinder such that the subject can now see the reward when approaching. Subjects that show high levels of motor selfregulation are expected to detour to one of the open ends of the cylinder as previously learned without first touching the front of the apparatus. MacLean et al. (2014) have used the cylinder task to investigate motor self-regulation (not self-control as they claimed; see above (Bari \& Robins 2013; Beran 2015)) using a broad comparative approach. The focus of the MacLean et al. study, as with other studies applying the cylinder task, was on primate, canine and bird species (Marshall-Pescini et al. 2015; Fagnani et al. 2016; Kabadayi et al. 2016; Vernouillet et al. 2016). The investigators found levels of motor self-regulation above $90 \%$ in the great apes and some social corvids, whereas performance was below $50 \%$ in many other bird and mammal species, including primates.

To broaden the range of species for a comprehensive discussion of behavioural inhibition, motor self-regulation was evaluated using the cylinder task in an ungulate species, the domestic goat. Feral and domestic goats have been shown to live in fission-fusion societies (Stanley \& Dunbar 2013; Ævarsdóttir 2014) and show various features related to complex cognition, such 109 as learning to learn (Langbein et al. 2008), categorization (Meyer et al. 2012), inferential reasoning (Nawroth et al. 2014), object permanence (Nawroth et al. 2015a) and learning of 111 complex two-step tasks (Briefer et al. 2014). In this study, goat capacity for motor self- 
112 regulation was evaluated as well as whether goats show motor self-regulation spontaneously or

113 learn to inhibit a prepotent response. While the experimental setup was identical in most

114 respects to the one used by MacLean et al. (2014), it was also investigated whether short,

115 distinct pointing gestures made by the experimenter during baiting would influence the side of

116 the cylinder to which the goats detoured. Although two studies have shown that goats

117 understand human gestures as an indication of a hidden reward (Kaminski et al. 2005; Nawroth

118 et al. 2015b, 2016a), their understanding of distal human gestures has yet to be demonstrated. As

119 there is some evidence that the lateralization of the brain underlies side biases in detour-reaching

120 tasks (Vallortigara \& Bisazza 2002; Reddon \& Hurd 2009), it was investigated whether the

121 goats would show a side bias in detouring the cylinder. Give their high level of sociability and

122 their strong performance in a variety of cognitive tasks, goats can be expected to show a high

123 level of motor self-regulation. According to the 'grazer-browser continuum' proposed by

124 Hofmann (1989), the goat is classified as an 'intermediate grazer' that feeds on a mixture of

125 shrubs/herbs/forbs and grass (Stuth 1991), which might favour a high level of motor self-

126 regulation to inhibit feeding on low-quality food in favour of searching for high-quality food.

127

128 Animals, materials \& methods

129 Ethics statement

130 All animal care and experimental procedures were performed in accordance with the

131 German welfare requirements for farm animals and the ASAB/ABS Guidelines for the Use of

132 Animals in Research (Anonymous 2016). All procedures involving animal handling and

133 treatment were approved by the Committee for Animal Use and Care of the Ministry of 
134 Agriculture, Environment and Consumer Protection of the federal state of Mecklenburg-

135 Vorpommern, Germany (Ref. No. 7221.3-2-012/15 and 7221.3-2-011/16).

136

137 Subjects and management conditions

The experiment was conducted with 22 female Nigerian dwarf goats (Capra aegagrus

hircus) between April and June in $2015(\mathrm{n}=10)$ and $2016(\mathrm{n}=12)$. The goats were bred and housed at the Leibniz Institute for Farm Animal Biology (FBN, Dummerstorf, Germany). At the beginning of the experiment, all of the goats were between 15 and 22 months (mean, 17 months). The animals were group-housed indoors. Their pen $(3 \times 4 \mathrm{~m})$ contained straw bedding and was equipped with an automatic waterer. The goats had ad libitum access to hay and were not foodrestricted during any phase of the experiment. They were maintained under a photoperiod of $12 \mathrm{~h}$

147 discrimination learning using a fully automated learning device at the age of six months. The goats also underwent open-field, novel-object and maze tests at that time (unpublished data) and were thus already habituated to human handling before the start of the detour-reaching experiment.

\section{General aspects of training and testing}

The area for testing the goats was located in the same building as the holding pen and comprised several compartments: a waiting area, a start box, the experimental area, and two return alleys (Fig. 1). The walls of the experimental area and the return alleys were $1.6 \mathrm{~m}$ in 
156 height and made of brown plywood. The doors to and from the experimental area were operated

157 remotely. The floor was covered with black rubber mats.

158 For habituation, the goats were moved as a group to the waiting area and were allowed to

159 enter the experimental area by passing through the open start box once a day for 4 consecutive 160 days. They freely explored the experimental area and the return alleys as a group for $30 \mathrm{~min}$ each 161 day. Training and testing were conducted in sessions from 9:00 to 11:00 and 13:00 to 15:00 from 162 Monday to Friday. For each session, the group was moved from the home pen to the waiting area. For each trial, an individual goat was gently pushed into the start box $(1 \times 1 \times 1 \mathrm{~m})$ by experimenter 1 (E1). In the interest of standardizing the experimental conditions, the goat remained in the start box for $10 \mathrm{~s}$ before a transparent acrylic guillotine door was lifted to allow entry into the experimental area $(2.9 \times 1.4 \mathrm{~m})$.

After entering the experimental area, the subject was allotted $60 \mathrm{~s}$ to retrieve the reward, which was presented in the rear section of the experimental area (Fig. 1). A piece of uncooked pasta (penne) was used as a reward in all phases of the experiment (Nawroth et al. 2014). After the goat retrieved the reward or after $60 \mathrm{~s}$ elapsed without reward retrieval, the left or right door at the rear end of the experimental area was opened remotely, and the goat was led into one of the return alleys by experimenter 2 (E2) to independently re-join the group in the waiting area 173 via the left or right return alley. Within one session, each subject underwent two to four trials. 174 The order of testing within consecutive trials was randomized, and the side of the return alley 175 was counterbalanced for individual goats in consecutive trials. All trials during training and 176 testing were videotaped for subsequent coding of behaviour (Panasonic WVCP500, Tamron 177 13VG2811ASIR-SQ lens, EverFocus EDRHD-4H4 HD-CCTV Hybrid DVR). 


\section{Cylinder task}

Apparatus. For the cylinder task, an opaque (shaping and training) or otherwise identical transparent (test) cylinder $(20 \mathrm{~cm}$ in length, $17.2 \mathrm{~cm}$ in diameter, and $5 \mathrm{~mm}$ in thickness) was used. Each cylinder was open on both sides and mounted horizontally on a wooden platform (42 $\mathrm{cm}$ in height) (Fig. 2). The wooden platform was fixed to the ground to maintain the cylinder in place throughout the trials. During shaping only, two bowls $(8 \mathrm{~cm}$ diameter) were attached at the openings of the opaque cylinder to encourage the goats to explore the cylinder (Fig. 2A).

\section{Procedure}

Shaping. Shaping was conducted to habituate the subjects to being alone in the experimental area and to induce them to approach the cylinder to obtain a reward. In a total of 16 trials, E2 baited both external bowls (Fig. 2A) with one piece of pasta each and left the experimental area before the goat entered the start box. The goat was released into the experimental area after $10 \mathrm{~s}$. If the goat did not retrieve at least one reward within $60 \mathrm{~s}, \mathrm{E} 2$ entered the experimental area and offered the pasta by hand. Two goats were excluded from the experiment at this stage. One refused to feed on the pasta, and the other showed extreme signs of arousal upon being left alone in the experimental area. All of the remaining goats directly approached the cylinder and ate the pasta from both bowls by the end of shaping.

Training. During training, the animals should learn to retrieve the reward from inside the opaque cylinder (Fig. 2B). In the first five training trials, the pasta was placed at the left or right edge of the cylinder, whereas in the subsequent trials, the reward was placed in the middle of the cylinder. For baiting, E2 was standing behind the cylinder when the goat entered the start box. When the goat looked through the transparent door in the direction of the cylinder, E2 made a 
202 short, distinct pointing gesture with her left or right arm and placed the pasta in the cylinder (see

203 ESM_video1). The side from which E2 baited the cylinder was counterbalanced across trials and

204 pseudo-randomized so that the cylinder was not baited twice in succession from the same side.

205 Then, E2 left the experimental area. Each goat was released into the experimental area after $10 \mathrm{~s}$.

206 The criterion for admission to the test phase was successful retrieval of the reward within $60 \mathrm{~s}$ in

207 six consecutive trials. All but one animal fulfilled the criterion within 12 trials. One goat required

20816 trials to reach the criterion.

209 Test. The testing procedure was identical to the final training procedure with the exception 210 that the transparent cylinder was substituted for the opaque cylinder (Fig. 2C). The test consisted 211 of 10 trials per animal (see ESM_video2).

212

\section{Data scoring and analysis}

Behavioural coding was performed with the video footage using The Observer 12.0

215 (Noldus Information Technology, Wageningen, Netherlands). As two goats were excluded from the experiment during shaping (see above), the data from 20 goats were analysed. For the test

217 trials, the latency to retrieve the reward ('latency') and the accuracy of the approach to the cylinder ('accuracy') were recorded. 'Latency' was defined as the length of time from the first step of the goat into the experimental area to its touch of the reward. Trials were rated as accurate $(0)$ when the goat detoured to one side of the cylinder without touching the exterior of the cylinder. By contrast, trials were rated as inaccurate (1) when the goat tried to approach the reward directly by touching the front or back of the cylinder prior to retrieving the reward. In a study of human infants, Noland and Rodriguez (2011) argued that only those touches of a transparent surface that correspond to the reward's position behind it express inhibition errors. I 
225 rated a trial as incorrect only when the goat touched the cylinder near the reward and not when it

226 briefly explored the edge of the cylinder (see ESM_video2). For each trial, 'side' (left or right)

227 was recorded, defined as the side by which the goat detoured the cylinder, and 'TD-cyl' was

228 calculated, defined as the total duration over which the goat touched the exterior of the cylinder.

229 To assess inter-observer reliability, a second observer who was not involved in the study

230 recorded all of the behavioural data of the animals for $25 \%$ of the test trials. Cohen's kappa

231 indicated excellent agreement between coders across all recorded behavioural data $(\mathrm{k}=0.98, p<$

232 0.001; The Observer 12.0).

233 Statistical analyses were performed using the SAS System for Windows (SAS 9.4, TS

234 Level 1M3, 2012). Generalized linear mixed models (PROC GLIMMIX) fitted for binary data

235 were constructed to investigate the impact of test trial on 'accuracy' and the effect of the short

236 distinct pointing gesture made by the experimenter during baiting on 'side'. In the model, the

237 distribution of the appropriate response variable (binary distribution) and the link function

238 (logit) was specified, and a general Satterthwaite approximation was used for the degrees of

239 freedom of the denominator. Individual animal was treated as the subject for the repeated

240 statement and was considered in the factor trial. The effects of test trial on 'latency' and 'TD-

241 cyl' were investigated by conducting repeated-measures ANOVA using PROC MIXED.

242 Individual animal was treated as subject for the repeated statement and was considered in the

243 factor trial. Least-squares means (LSM) and their standard errors (SE) were calculated for the

244 variables of interest in all models. Where significant main effects were found $(p<0.05)$,

245 adjustments for multiple testing were applied (Tukey-Kramer correction) in subsequent multiple

246 comparison procedures. The binomial test was employed to detect individual side biases in 
247 detouring the cylinder. In addition, a continuous laterality index was calculated for each subject

248 to test its correlation with 'accuracy' in test trials (Hopkins 1999).

249

250

\section{Results}

251

The average accuracy during the test was $62.5 \%( \pm 10.86 \%)$. There was no impact of trial

252

253

254

255

256

257

258

259

260

261

262

263

264

265

266

267

268

number on accuracy $\left(F_{9,171}=0.85\right.$, n.s. $)$. The accuracy of the individuals was variable across trials (Fig. 3). While nine goats detoured the cylinder correctly in seven or more trials, 11 animals touched the exterior of the cylinder in approximately every other trial.

Mean contact time with the cylinder (TD-cyl) in the test was $1.28 \mathrm{~s}( \pm 0.64 \mathrm{~s})$. There was no impact of trial number on TD-cyl $\left(F_{9,171}=0.93\right.$, n.s. $)$. In most of the inaccurate trials, goats started detouring towards one of the open ends of the cylinder but then changed direction and briefly touched the near side of the cylinder in the region of the reward before finally detouring to the side and retrieving the reward (see ESM_video2).

In contrast to its impact on TD-cyl, trial number had a significant impact on latency to retrieve the reward in the test $\left(F_{9,134}=7.85, p<0.001\right)$ (Fig. 4$)$. Latency was $34 \mathrm{~s}( \pm 3.62 \mathrm{~s})$ in trial one and decreased to below 10 s from trial four onwards. Pairwise comparisons revealed latency in the test to be longer in trial one than in all subsequent trials (all $p<0.05$ ).

Furthermore, latency was longer in the first test trial than in the last training trial $(p<0.01)$.

A short, distinct pointing gesture made by the experimenter during baiting had no effect on the side (left/right) by which the goats detoured the cylinder to retrieve the reward $\left(F_{1,151}=0.22\right.$, n.s). Ten out of 20 subjects $(50 \%)$ showed an individual side bias in the test $(p<0.05)$. Among these subjects, six had a preference for detouring the cylinder to the left, and four preferentially 
269 detoured to the right. There was no correlation between the index of laterality and the level of

270 accuracy in the test (Spearman rank correlation $\left(\mathrm{r}_{\mathrm{S}}\right)=-0.10, p=0.66, \mathrm{n}=20$ ).

271

272 Discussion

The goats in this study were able to retrieve a reward by showing motor self-regulation at a

level comparable to or better than that of many other mammal and bird species tested to date (MacLean et al. 2014, Support. Inform., Table_S05; Vernouillet et al. 2016). Only great apes, some social corvids and various canine species have shown fundamentally better performance than the studied goats in the cylinder task (MacLean et al. 2014; Fagnani et al. 2016; Kabadayi et and performance in the cylinder task for 25 previously studied mammal species and the goat, the value for goat lies slightly below the regression line (Fig. 5). Unfortunately, to date, almost exclusively primate and canine species have been examined using this task. It would be of particular interest to study other taxa; for example, it would be of interest to study several herbivorous species to determine the effects of different feeding strategies or different social systems on their levels of behavioural control.

It has been demonstrated that monkey species living in fission-fusion societies, which are based on individual recognition, social cooperation and pair bonding, tend to show higher levels of motor self-regulation than do species living in more stable groups (Amici et al. 2008). Feral and domestic goats have been shown to live in fission-fusion societies (Stanley \& Dunbar 2013; Evarsdóttir 2014), to generate individual contact calls in both kids and their mothers for individual recognition (Briefer \& McElligott 2011; Briefer et al. 2012), and to easily

291 discriminate between members of their own group and those of a different group (Keil et al. 
292 2012). According to its feeding ecology, the goat is classified as an 'intermediate grazer',

293 meaning it exhibits a marked degree of foraging selectivity (Stuth 1991). These aspects of the

294 goat's social life, cognitive abilities and feeding ecology may explain its apparently good

295 performance in motor-self regulation. However, I found large inter-individual variation among

296 animals in the level of motor self-regulation. Only approximately half of the subjects exhibited

297 high levels of behavioural inhibition. Nine out of the 20 goats were able to consistently suppress

298 the prepotent response of directly approaching the visible reward in seven or more trials, whereas

299 the remainder exhibited poor motor control. These latter goats approached the reward directly in

300 approximately every other trial. There was no improvement of motor self-regulation at the group

301 level over the testing period.

302 Previous studies using the cylinder task have reported inconsistent results regarding the 303 learning of motor self-regulation. While MacLean et al. (2014), who compared motor self304 regulation in 36 species, did not report data on improvement over test trials, other authors have 305 analysed performance across trials to evaluate potential learning effects. No such effects on motor self-regulation were found in some studies of dogs and wolves (Bray et al. 2014; Marshall-Pescini et al. 2015). By contrast, trial number was found to have an effect on motor-self regulation in dogs in one study and in some bird species, indicating that learning across trials occurred (Fagnani et al. 2016; Kabadayi et al. 2016; Vernouillet et al. 2016). Most recent studies have compared the level of accuracy between only the first and last blocks of five trials. It has been suggested that an improvement in motor self-regulation across trials might indicate insufficient training with the opaque cylinder (Santos et al. 1999; Smith et al. 1999) or a lack of experience with transparent surfaces (Yates and Bremner 1988; Vernouillet et al. 2016);

314 however, no such relation was found by Fagnani et al. (2016) in dogs. Both explanations can be 
315 excluded for the goats in this study. They performed well with the opaque cylinder after only a

316 few training trials, and they had experience with transparent surfaces, including those in a prior

317 maze test (see Animals, materials \& methods) and the transparent doors in this study (see Fig. 1).

318 Furthermore, nearly all of the goats accurately detoured the cylinder in $50 \%$ of all trials in the

319 test, indicating that any inaccurate trials did not result from a lack of knowledge about how to

320 solve the task or the concept of transparency. It seems that for the majority of the goats, the

321 visual salience of the reward inside the transparent cylinder was sufficiently strong to overpower

322 the previously learned accurate motor pattern used to retrieve the reward. Some researchers have

323 argued that the visibility of the reward behind the transparent barrier acts as a "magnet for

324 perception", making it very difficult for animals to avoid direct approach and activate the learned

325 behaviour pattern of detouring (Vallortigara and Regolin 2002). Similar effects have been shown

326 in various object retrieval tasks in human infants at the age of 7 months (Diamond 1981;

327 Diamond 1990). Therefore, errors during test trials appear to result from failure of reliable motor

328 self-regulation. However, I cannot exclude the possibility that the accuracy of the goats in the

329 test would have improved after more trials. In a study with song sparrows, some individuals

330 reached the learning criterion of six correct trials in succession in the cylinder task only after 50

331 trials (Boogert et al., 2011). However, this outcome is unrelated to behaviour control; rather, it

332 shows that some sparrows learned a new behaviour pattern that allowed them to overcome the 333 impulse to directly approach the reward.

334 Unfortunately, only one other study on motor self-regulation has been performed in which 335 latency to retrieve the reward was investigated; the subjects were various parrot species 336 (Kabaday et al. 2017). According to the authors of that study, a reduction in latency is generally 337 seen as a sign of learning the task. In the current study, the trial number in the test had a 
338 significant effect on the latency to retrieve the reward. However, this effect was mainly caused 339 by the latency in the first test trial, which was significantly longer than that of any of the 340 subsequent trials. Additionally, latency was significantly longer in the first test trial than in the

341 final training trial. The goats reacted with great caution to the introduction of the transparent 342 cylinder in the test. Novel objects are known to be fear-inducing stimuli in ungulates (Désiré et 343 al. 2004). Therefore, I believe that the goats' initial fear of the transparent cylinder rather than a 344 learning effect was responsible for reducing latency over the first trials. Owing to this fear of 345 new objects, one would expect animals performing the cylinder task to carefully approach and 346 investigate the exterior of the transparent cylinder before attempting to retrieve the reward inside, 347 especially in the first few trials. Therefore, initial fear could indirectly affect the number of 348 inaccurate trials during the test. This possibility is important to consider in future detour-reaching 349 studies. However, the goats in this study rapidly overcame their initial fear of approach as 350 indicated by the rapid decrease in latency. Additionally, as discussed above, the number of 351 inaccurate trials was not affected by the trial number.

352 Detour tasks are widely accepted as the most suitable tasks for comparing motor self-regulation 353 across species. However, recently, there has been increasing criticism of the general validity of 354 this task resulting from the large variation among species and the large inconsistency in 355 performance among different detour tasks within species (Kabadayi et al., 2017; Kabadayi et al., 356 2018; van Horik et al., 2018). Individual performance in detour tasks, especially the cylinder 357 task, may be confounded by various accompanying factors, such as the level of neophobia of 358 novel objects (Regolin et al. 1994), level of experience with transparent surfaces as barriers and 359 learning effects. Although I have discussed the influences of some of these factors in detail, it 360 would be valuable to investigate the specific aspects of various detour tasks within the 
361 framework of task batteries, as has been done in some recent studies (Amici et al., 2008; Brucks

362 et al. 2017).

363 There is ongoing debate as to whether and how animals perceive and process human-given

364 social cues, such as pointing, to indicate the location of a reward or to direct an animal's

365 movement (Tauzin et al. 2015). Among domestic animals, dogs (Pongracz et al. 2013) and

366 various farm animals, such as goats (Kaminski et al. 2005; Nawroth et al. 2015b) and horses

367 (Proops et al. 2010; Lovrovich et al. 2015), have been shown to make use of human-given cues.

368 However, one should not overestimate the cognitive abilities necessary to respond appropriately

369 to human pointing, as most experiments involving human pointing did not differentiate between

370 local or stimulus enhancement and actual referential comprehension of the task. For monkeys

371 and horses, pointing worked well when the pointing finger, hand or arm remained close to the

372 target until the animal made a choice (Maros et al. 2008; Schmitt et al. 2014). A more

373 challenging form of human social cues is momentary pointing from a distance (Gácsi et al.

374 2009). In the present study, the experimenter made only a short, distinct pointing gesture to

375 indicate the side from which the cylinder was baited before the subject was allowed to make a

376 choice. Dogs and elephants have been shown to be capable of using social cues as referential

377 signals during momentary pointing (Pongracz et al. 2013; Smet \& Byrne 2014). With the goats

378 in the present study, there was no evidence that the short, distinct pointing gesture during baiting

379 had an impact on the side by which the goats detoured the cylinder. Therefore, I do not believe

380 that the pointing gestures had any influence on the level of motor self-regulation in this study.

381 Different types of detour tests, aside from those studying motor self-regulation, have been

382 employed to investigate lateralization in several species under natural and experimental

383 conditions (Vallortigara et al. 1999; Baragli et al. 2011; Leliveld et al. 2013; Siniscalchi et al. 
384 2013). Laterality refers to the phenomenon in which external attractions are perceived and

385 processed differently by the two cerebral hemispheres depending on their novelty and emotional

386 value and in which the execution of motor behaviour is preferentially performed by one side of

387 the body. Owing to lateral eye position and the decussation of optic nerve fibres at the optic

388 chiasm in ungulates (approximately 80-90\% in large domestic ungulates (Shamir \& Ofri 2008)),

389 visual cues perceived by the left eye are largely, though not exclusively, processed by the right

390 hemisphere and vice versa. In a recent study in goats (Nawroth et al. 2016b), the authors did not

391 find agreement over repeated trials regarding the side to which goats detoured a transparent

392 barrier. By contrast, half of the animals in this study showed individual side biases in detouring

393 the transparent cylinder in the test. An approximately equal number of goats preferred either

394 side. This result indicates that at least some of the goats showed lateralization of the detour

395 behaviour at the individual level, but such lateralization was not evident at the population level.

396 Similar results have been found regarding lateralization of detour behaviour in sheep (Versace et

397 al. 2007). However, there was no impact of laterality on the level of motor self-control in goats 398 in this study.

400 Conclusions

The results demonstrate that goats display motor self-regulation at a level comparable to or 402 better than the levels observed in many other bird and mammal species tested to date. However, the goats did not show any improvement in the level of motor self-regulation across trials and 404 showed large intra- and inter-individual variability across test trials. The results indicate the 405 importance of considering individual reaction patterns when analysing detour behaviour. 


\section{Acknowledgements}

408 I would like to thank Katrin Siebert for data coding, Armin Tuchscherer for statistical 409 advice, and Dieter Sehland and Heinz Deike for excellent technical assistance. I would also like 410 to thank Christian Nawroth for in-depth discussions and valuable comments on an earlier version 411 of the manuscript. Finally, we thank Lucia Regolin and two other anonymous reviewers for their 412 helpful comments on an earlier version of this manuscript.

\section{References}

Ævarsdóttir H. 2014. The secret life of Icelandic goats: activity, group structure and plant selection of the Icelandic goatMaster Thesis. Faculty of Life and Environmental Science, University of Iceland.

Amici F, Aureli F, Call J. 2008. Fission-fusion dynamics, behavioral flexibility, and inhibitory control in primates. Current Biology 18:1415-1419.

Anderson JR, Kuroshima H, Fujita K. 2010. Delay of gratification in Capuchin Monkeys (Cebus apella) and Squirrel Monkeys (Saimiri sciureus). Journal of Comparative Psychology 124:205-210.

Anonymous. 2016. Guidelines for the treatment of animals in behavioural research and teaching. Animal Behaviour 111:I-XI.

Ballarin C, Povinelli M, Granato A, Panin M, Corain L, Peruffo A, and Cozzi B. 2016. The Brain of the Domestic Bos taurus: Weight, Encephalization and Cerebellar Quotients, and Comparison with Other Domestic and Wild Cetartiodactyla. PLoS ONE 11:e0154580.

Baragli P, Vitale V, Paoletti E, Sighieri C, Reddon AR. 2011. Detour behaviour in horses (Equus caballus). Journal of Ethology 29:227-234. 
430 Bari A, Robbins T. 2013. Inhibition and impulsivity: Behavioral and neural basis of response

431

432

433

434

435

436

437

438

439

440

441

442

443

444

445

446

447

448

449

450

451

452 control. Progress in Neurobiology 108:44-79.

Beran MJ. 2015. The comparative science of "self-control": what are we talking about? Frontiers in Psychology 6:1-4.

Bond AB, Kamil AC, Balda RP. 2007. Serial reversal learning and the evolution of behavioral flexibility in three species of North American corvids (Gymnorhinus cyanocephalus, Nucifraga columbiana, Aphelocoma californica). Journal of Comparative Psychology $121: 372-379$

Boogert NJ, Anderson RC, Peters S, Searcy WA, Nowicki S. 2011. Song repertoire size in male song sparrows correlates with detour reaching, but not with other cognitive measures. Animal Behaviour 81:1209-1216.

Bray EE, MacLean EL, Hare BA. 2014. Context specificity of inhibitory control in dogs. Animal Cognition 17:15-31.

Briefer E, McElligott AG. 2011. Mutual mother-offspring vocal recognition in an ungulate hider species (Capra hircus). Animal Cognition 14:585-598.

Briefer EF, Padilla de la Torre M, McElligott AG. 2012. Mother goats do not forget their kids' calls. Proc Biol Sci 279:3749-3755.

Briefer EF, Haque S, Baciadonna L, McElligott AG. 2014. Goats excel at learning and remembering a highly novel cognitive task. Frontiers in Zoology 11:20.

Brucks D, Marshall-Pescini S, Wallis LJ, Huber L, and Range F. 2017. Measures of Dogs' Inhibitory Control Abilities Do Not Correlate across Tasks. Frontiers in Psychology 8:849.

Burke WH, Zencius AH, Wesolowski MD, Doubleday F. 1991. Improving executive function disorders in brain-injured clients. Brain Injury 5:241-252. 
453 Chappell J. 2017. Bird brains: Does absolute size matter? Learning \& Behavior 45:1-2.

454 Désiré L, Veissier I, Despres G, Boissy A. 2004. On the way to assess emotions in animals: Do $455 \quad$ lambs (Ovis aries) evaluate an event through its suddenness, novelty, or unpredictability? 456 Journal of Comparative Psychology 118:363-374.

457 Diamond A. 1981. Retrieval of an object from an open box: the development of visual-tactile $458 \quad$ control of reaching in the frst year of life. Soc Res Child Dev 3:78.

459 Diamond A. 1990. Developmental time course in human infants and infant monkeys, and the neural bases of, inhibitory control in reaching. Annals of the New York Academy of Sciences 608:637-676.

Diamond A. 2013. Executive functions. Annual Review of Psychology 64:135-168.

463 Evans T, Perdue B, Parrish A, Beran MJ. 2014. Working and waiting for better rewards:Selfcontrolin two monkey species (Cebus apella and Macaca mulatta). Behavioural Processes $103: 236-242$

466

467

468

469

470

471

472

473

474

Fagnani J, Barrera G, Carballo F, Bentosela M. 2016. Is previous experience important for inhibitory control? A comparison between shelter and pet dogs in A-not-B and cylinder tasks. Animal Cognition 19:1165-1172.

Gácsi M, McGreevy P, Kara E, Miklosi A. 2009. Effects of selection for cooperation and attention in dogs. Behav Brain Funct 5:1-8.

Hillemann F, Bugnyar T, Kotrschal K, Wascher CAF. 2014. Waiting for better, not for more: corvids respond to quality in two delay maintenance tasks. Animal Behaviour 90:1-10.

Hofmann RR. 1989. Evolutionary steps of ecophysiological adaptation and diversification of ruminants - A comparative view of their digestive-system. Oecologia 78:443-457. 
475 Hopkins WD. 1999. On the other hand: Statistical issues in the assessment and interpretation of 476 hand preference data in nonhuman primates. International Journal of Primatology 20:851866.

478 Jelbert SA, Taylor AH, Gray RD. 2016. Does absolute brain size really predict self-control? Hand-tracking training improves performance on the A-not-B task. Biology Letters 12:20150871.

481

482

483

484

485

486

487

488

489

490

491

492

493

494

495

Kabadayi C, Taylor LA, von Bayern AM, Osvath M. 2016. Ravens, New Caledonian crows and jackdaws parallel great apes in motor self-regulation despite smaller brains. Royal Society Open Science 3:160104.

Kabadayi C, Krasheninnikova A, O'Neill L, van de Weijer J, Osvath M, and von Bayern AMP. 2017. Are parrots poor at motor self-regulation or is the cylinder task poor at measuring it? Animal Cognition 20:1137-1146.

Kabadayi C, Bobrowicz K, and Osvath M. 2018. The detour paradigm in animal cognition. Animal Cognition 21:21-35.

Kaminski J, Riedel J, Call J, Tomasello M. 2005. Domestic goats, Capra hircus, follow gaze direction and use social cues in an object choice task. Animal Behaviour 69:11-18.

Keil NM, Imfeld-Mueller S, Aschwanden J, Wechsler B. 2012. Are head cues necessary for goats (Capra hircus) in recognising group members? Animal Cognition 15:913-921.

Kralik JD, Hauser MD, Zimlicki R. 2002. The relationship between problem solving and inhibitory control: Cotton-top tamarin (Saguinus oedipus) performance on a reversed contingency task. Journal of Comparative Psychology 116:39-50. 
496 Langbein J, Siebert K, Nuernberg G. 2008. Concurrent recall of serially learned visual

497

498

499

500

501

502

503

504

505

506

507

508

509

510

511

512

513

514

515

516

517

518

discrimination problems in dwarf goats (Capra hircus). Behavioural Processes 79:156164.

Leliveld LM, Langbein J, Puppe B. 2013. The emergence of emotional lateralization: Evidence in non-human vertebrates and implications for farm animals. Applied Animal Behaviour Science 145:1-14.

Lovrovich P, Sighieri C, Baragli P. 2015. Following human-given cues or not? Horses (Equus caballus) get smarter and change strategy in a delayed three choice task. Applied Animal Behaviour Science 166:80-88.

MacLean EL, Hare B, Nunn CL, Addessi E, Amici F, Anderson RC, Aureli F, Baker JM, Bania AE, Barnard AM, Boogert NJ, Brannon EM, Bray EE, Bray J, Brent LJN, Burkart JM, Call J, Cantlon JF, Cheke LG, Clayton NS, Delgado MM, DiVincenti LJ, Fujita K, Herrmann E, Hiramatsu C, Jacobs LF, Jordan KE, Laude JR, Leimgruber KL, Messer EJE, Moura ACD, Ostojic L, Picard A, Platt ML, Plotnik JM, Range F, Reader SM, Reddy RB, Sandel AA, Santos LR, Schumann K, Seed AM, Sewall KB, Shaw RC, Slocombe KE, Su YJ, Takimoto A, Tan JZ, Tao R, van Schaik CP, Viranyi Z, Visalberghi E, Wade JC, Watanabe A, Widness J, Young JK, Zentall TR, Zhao YN. 2014. The evolution of self-control. Proceedings of the National Academy of Sciences of the United States of America 111:E2140-E2148.

Maclean EL, Sandel AA, Bray J, Oldenkamp RE, Reddy RB, Hare BA. 2013. Group Size Predicts Social but Not Nonsocial Cognition in Lemurs. PLoS ONE 8:e66359.

Manrique MH, Call J. 2015. Age-dependent cognitive inflexibility in great apes. Animal Behaviour 102:1-6. 
519 Maros K, Gacsi M, Miklosi A. 2008. Comprehension of human pointing gestures in horses $520 \quad$ (Equus caballus). Animal Cognition 11:457-466.

521 Marshall-Pescini S, Viranyi Z, Range F. 2015. The effect of domestication on inhibitory control:

$522 \quad$ wolves and dogs compared. PLoS ONE 10:e 0118469.

523 Meyer S, Nuernberg G, Puppe B, Langbein J. 2012. The cognitive capabilities of farm animals: 524 categorisation learning in dwarf goats (Capra hircus). Animal Cognition 15:567-576.

525 Miyake A, Friedman N, Emerson M, Witzki A, Howerter A, Wagner T. 2000. The unity and 526 diversity of executive functions and their contributions to complex 'Frontal Lobe' tasks: a 527 latent variable analysis. Cognitive Psychology 41:49-100.

528 Nawroth C, von Borell E, Langbein J. 2014. Exclusion performance in dwarf goats (Capra $529 \quad$ aegagrus hircus) and sheep (Ovis orientalis aries). PLoS ONE 9:e93534.

530 Nawroth C, von Borell E, Langbein J. 2015a. Object permanence in the dwarf goat (Capra 531 aegagrus hircus): Perseveration errors and the tracking of complex movements of hidden 532 objects. Applied Animal Behaviour Science 167:20-26.

533 Nawroth C, von Borell E, Langbein J. 2015b. 'Goats that stare at men': dwarf goats alter their 534 behaviour in response to human head orientation, but do not spontaneously use head 535 direction as a cue in a food-related context. Animal Cognition 18:65-73.

536 Nawroth C, von Borell E, Langbein J. 2016a. 'Goats that stare at men' - revisited: do dwarf goats 537 alter their behaviour in response to eye visibility and head direction of a human? Animal 538 Cognition 19:667-672.

539 Nawroth C, Baciadonna L, McElligott AG. 2016b. Goats learn socially from humans in a spatial $540 \quad$ problem-solving task. Animal Behaviour 121:123-129. 
541 Noland JS, Rodrigues NP. 2012. Direct touches to clear barriers: developmental sensitivity of a

542 new measure of the production of ineffective responses in infancy. Child Neuropsychol $543 \quad 18: 506-511$.

544 Osthaus B, Proops L, Hocking I, Burden F. 2012. Spatial cognition and perseveration by horses, 545 donkeys and mules in a simple A-not-B detour task. Animal Cognition 16:301-305.

546 Pongracz P, Gacsi M, Hegedüs D, Peter A, Miklosi A. 2013. Test sensitivity is important for 547 detecting variability in pointing comprehension in canines. Anim Cogn 16:721-735.

548 Pongracz P, Miklosi A, Kubinyi E, Gurobi K, Topal J, Csanyi V. 2001. Social learning in dogs:

549 the effect of a human demonstrator on the performance of dogs in a detour task. Animal 550 Behaviour 62:1109-1117.

551 Proops L, Walton M, McComb K. 2010. The use of human-given cues by domestic horses, 552 Equus caballus, during an object choice task. Animal Behaviour 79:1205-1209.

553 Reddon AR, Hurd PL. 2009. Individual differences in cerebral lateralization are associated with 554 shy-bold variation in the convict cichlid. Animal Behaviour 77:189-193.

Regolin L, Vallortigara G, Zanforlin M (1994) Perceptual and motivational aspects of detour behaviour in young chicks. Animal Behaviour 47(1):123-131.

Santos LR, Ericson BN, Hauser MD. 1999. Constraints on problem solving and inhibition: Object retrieval in cotton-top tamarins (Saguinus oedipus oedipus). Journal of Comparative Psychology 113:186-193.

Schmitt V, Schloegl C, Fischer J. 2014. Seeing the Experimenter Influences the Response to Pointing Cues in Long-Tailed Macaques. PloS One 9:e91348 veterinary ophthalmology. Oxford: Blackwell Publishing, 445-462. 
564 Siniscalchi M, Pergola G, Quaranta A. 2013. Detour behaviour in attack-trained dogs: left565 turners perform better than rightturners. Laterality 18:282-293.

566 Smet AF, Byrne RW. 2014. Interpretation of human pointing by African elephants: 567 generalisation and rationality. Animal Cognition 17:1365-1374.

568 Smith LB, Thelen E, Titzer R, McLin D. 1999. Knowing in the context of acting: The task 569 dynamics of the A-not-B error. Psychological Review 106:235-260.

570 Stanley CR, Dunbar RIM. 2013. Consistent social structure and optimal clique size revealed by 571 social network analysis of feral goats, Capra hircus. Animal Behaviour 85:771-779.

572 Stuth JW. 1991. Foraging behavior. In: Heitschmidt RK, Stuth JW, eds. Grazing Management: 573 An Ecological Perspective. Portland Oregon: Timber Press, 259-272.

574 Tapp PD, Siwak CT, Estrada J, Head E, Muggenburg BA, Cotman CW, Milgram NW. 2003. 575 Size and reversal learning in the beagle dog as a measure of executive function and $576 \quad$ inhibitory control in aging. Learning and Memory 10:64-73.

577 Tauzin T, Csík A, Kis A, Topál J. 2015. What or Where? The Meaning of Referential Human $578 \quad$ Pointing for Dogs (Canis familiaris). Journal of Comparative Psychology 129:334-338.

579 Vallortigara G, Bisazza A. 2002. How ancient is brain lateralization? In: Rogers LJ, Andrew 580 RJ, eds. Comparative vertebrate lateralization. Cambridge: Cambridge University Press, $5819-69$.

582 Vallortigara G, Regolin L. 2002. Facing an obstacle: Lateralization of object and spatial 583 cognition. In: Rogers LJ, and Andrew R, eds. Comparative Vertebrate Lateralization. 584 Cambridge: Cambridge University Press, 383-444.

585 Vallortigara G, Regolin L, Pagni P. 1999. Detour behaviour, imprinting and visual lateralization 586 in the domestic chick. Cognitive Brain Research 7:307-320. 
587 van Horik JO, Langley EJG, Whiteside MA, Laker PR, Beardsworth CE, and Madden JR. 2018.

588 Do detour tasks provide accurate assays of inhibitory control? Proceedings of the Royal $589 \quad$ Society B: Biological Sciences 285: 20180150.

590 Vernouillet A, Anderson J, Clary D, Kelly DM. 2016. Inhibition in Clark's nutcrackers 591 (Nucifraga columbiana): results of a detour-reaching test. Animal Cognition 19:661-665.

592 Versace E, Morgante M, Pulina G, Vallortigara G. 2007. Behavioural lateralization in sheep 593 (Ovis aries). Behavioural Brain Research 184:72-80.

594 Vlamings PHJM, Hare B, Call J. 2010. Reaching around barriers: the performance of the great 595 apes and 3-5-year-old children. Animal Cognition 13:273-285.

596 Yates DJ, Bremner JG (1988) Conditions for Piagetian stage IV search errors in a task using 597 transparent occluders. Infant Behavior Development 11:411-417. 
599 Fig. 1. Experimental setup. Sketch showing the different compartments of the test area: waiting 600 area, start box, experimental area and two return alleys. The experimental area was video 601 monitored. All doors to and from the experimental area were operated remotely.

602

603

Fig. 2. Cylinders used in the different experimental phases. A) Shaping. B) Training. C) Test.

604

605

Fig. 3. Individual test trial accuracy. Accurate $(\operatorname{contact}=0)$ and inaccurate trials $(\operatorname{contact}=1)$

606 of individual goats in the testing phase.

607

608

Fig. 4. Latency to retrieve the reward. Mean latency $(s, L S M \pm S E)$ to retrieve the reward in 609 the test and in the final training trial. Significant differences between trials are indicated by asterisks $(* \mathrm{p}<0.05, * * \mathrm{p}<0.01)$.

611

612

Fig. 5. Relationship between brain size and motor self-regulation. Relationship between

613 absolute brain size and performance in the cylinder task for 26 mammal species from 4 orders. The trend line is based on a regression across all species. The two domestic animals species are marked in red. (1, Mongolian gerbil; 2, marmoset; 3, fox squirrel; 4, golden-headed lion tamarin; 5, mongoose lemur; 6 , black lemur; 7, ring-tailed lemur; 8, squirrel monkey; 9, brown lemur; 10, Coquerel's sifaka; 11, redbellied lemur; 12, ruffed lemur; 13, aye aye; 14, capuchin monkey; 15, coyote; 16, domestic dog; 17, 619 rhesus macaque; 18, golden snub-nosed monkey; 19, grey wolf; 20, domesticated goat; 21, hamadryus 620 baboon; 22, olive baboon; 23, bonobo; 24, chimpanzee; 25, orangutan; 26, gorilla [MacLean et al. 2014; 621 Ballarin et al. 2016]). 
Figure 1

Sketch of the area for testing the goats.

The sketch shows the different compartments of the test area: a waiting area, a start box, an experimental area and two return alleys. The experimental area was video monitored. All doors to and from the experimental area were operated remotely.

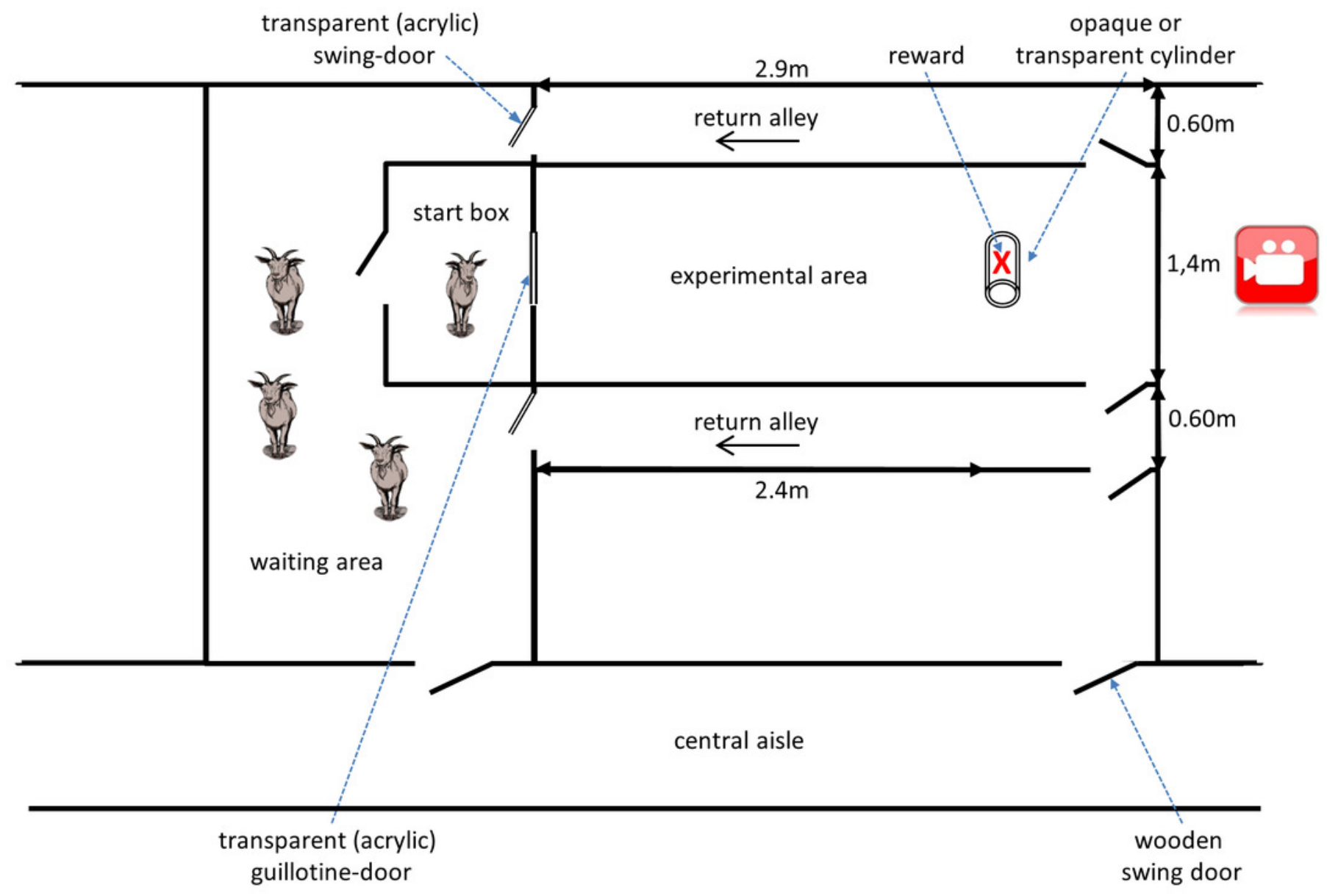




\section{Figure 2}

Cylinders used in the different experimental phases.

Cylinders used in the different experimental phases. A) Shaping. B) Training. C) Test.
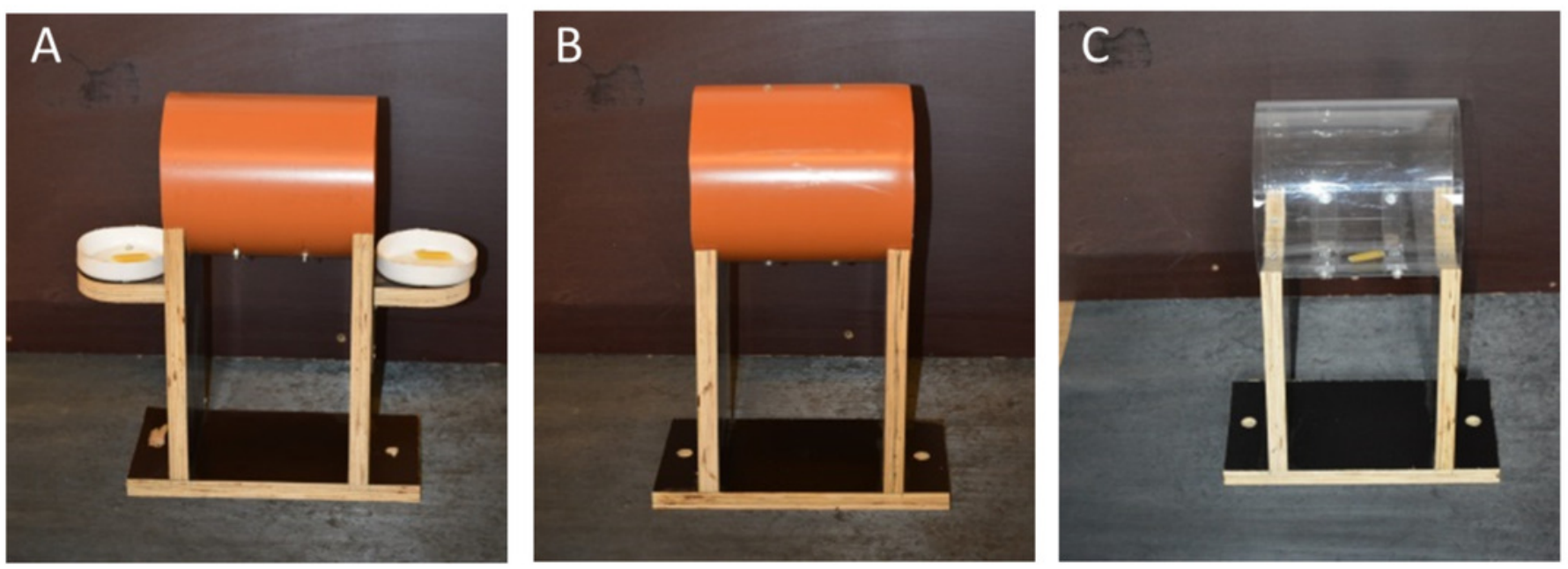


\section{Figure 3}

Individual test trial accuracy.

Individual test trial accuracy. Accurate (contact $=0$ ) and inaccurate trials (contact $=1$ ) of individual goats in the testing phase. Next to the number of the animal, each graph is marked with a different letter (from A to T). 

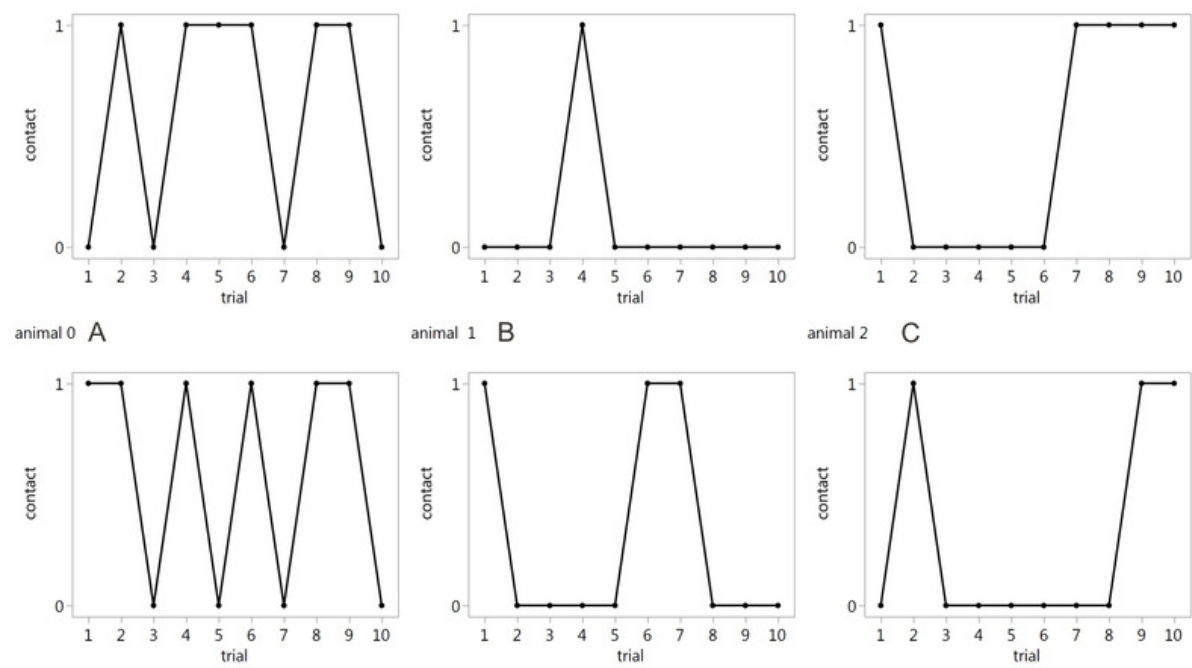

animal 1 B

animal $2 \mathrm{C}$
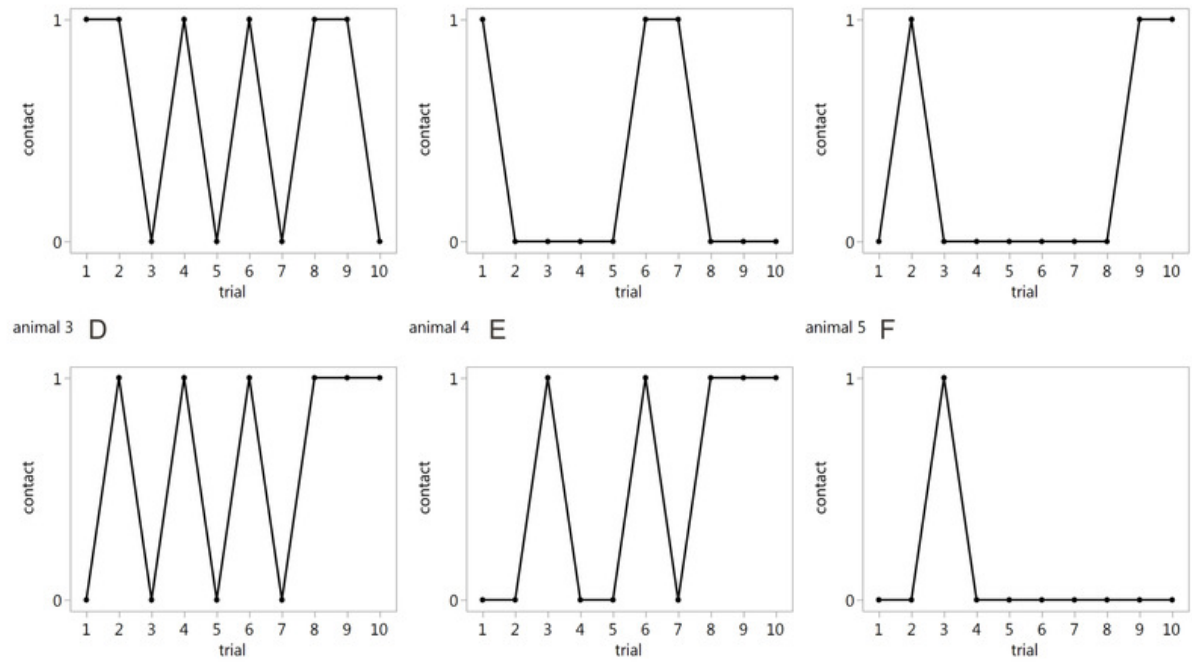
animal 4 E animal $5 \mathrm{~F}$
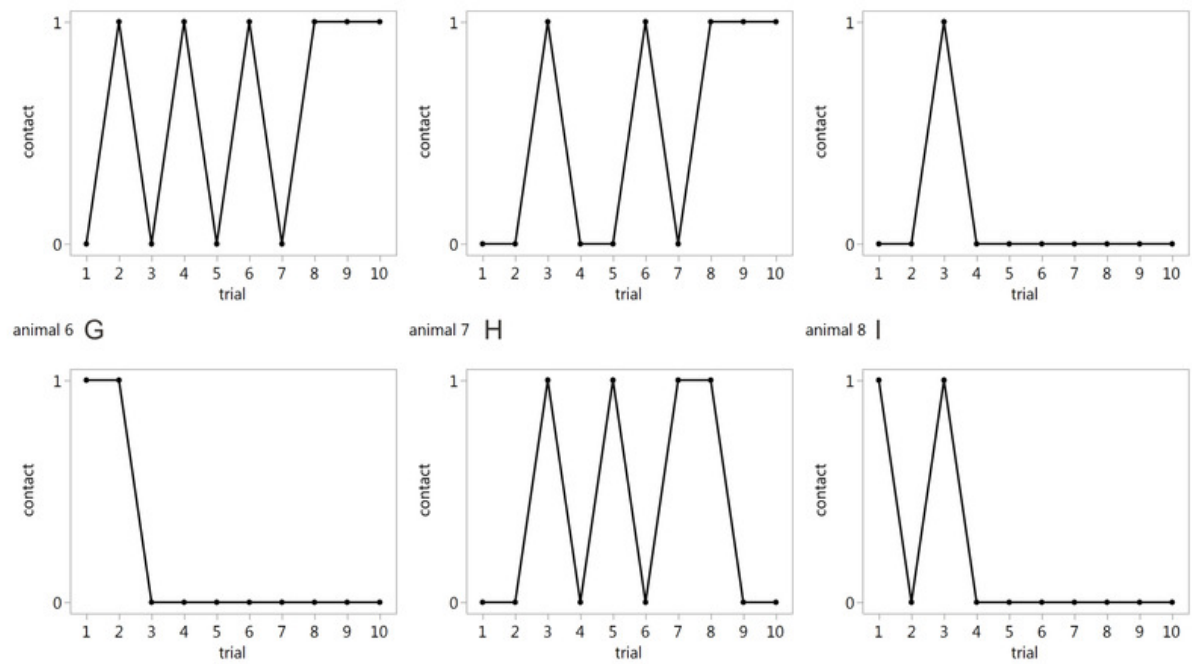

animal $7 \mathrm{H}$
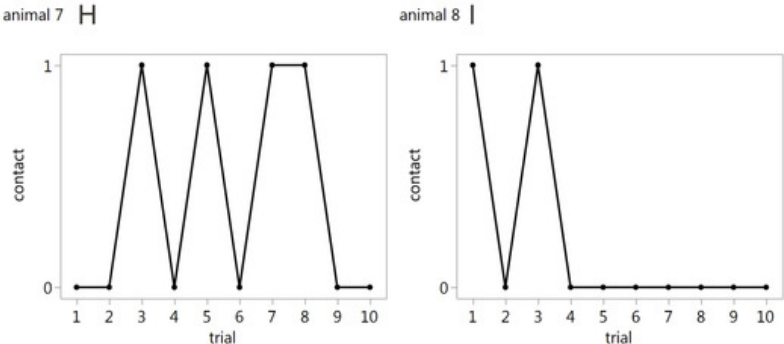

animal $9 \mathrm{~J}$

animal $10 \mathrm{~K}$

animal $11 \mathrm{~L}$
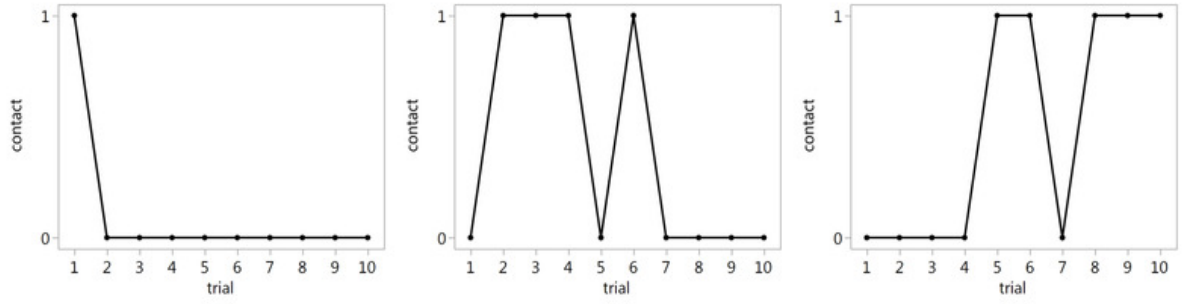

animal $12 \mathrm{M}$

animal $13 \mathrm{~N}$

animal $14 \mathrm{O}$
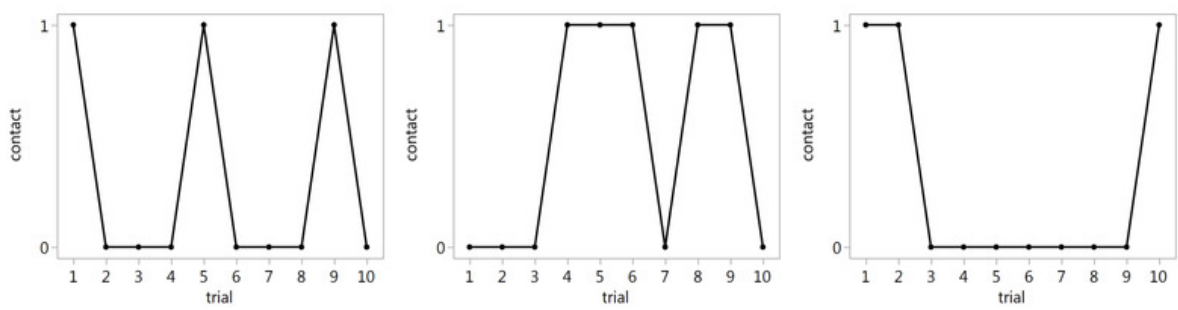

animal $15 \mathrm{P}$ animal $17 \mathrm{Q}$ animal $19 \mathrm{R}$
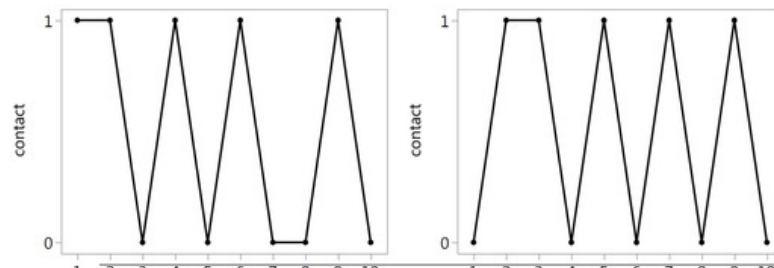

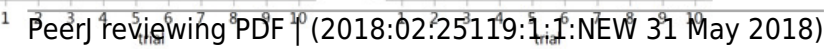


Figure 4

Latency to retrieve the reward.

Latency to retrieve the reward. Mean latency $(S, L S M \pm S E$ ) to retrieve the reward in the test and in the final training trial. Significant differences between trials are indicated by asterisks $(* p<0.05, * * p<0.01)$.

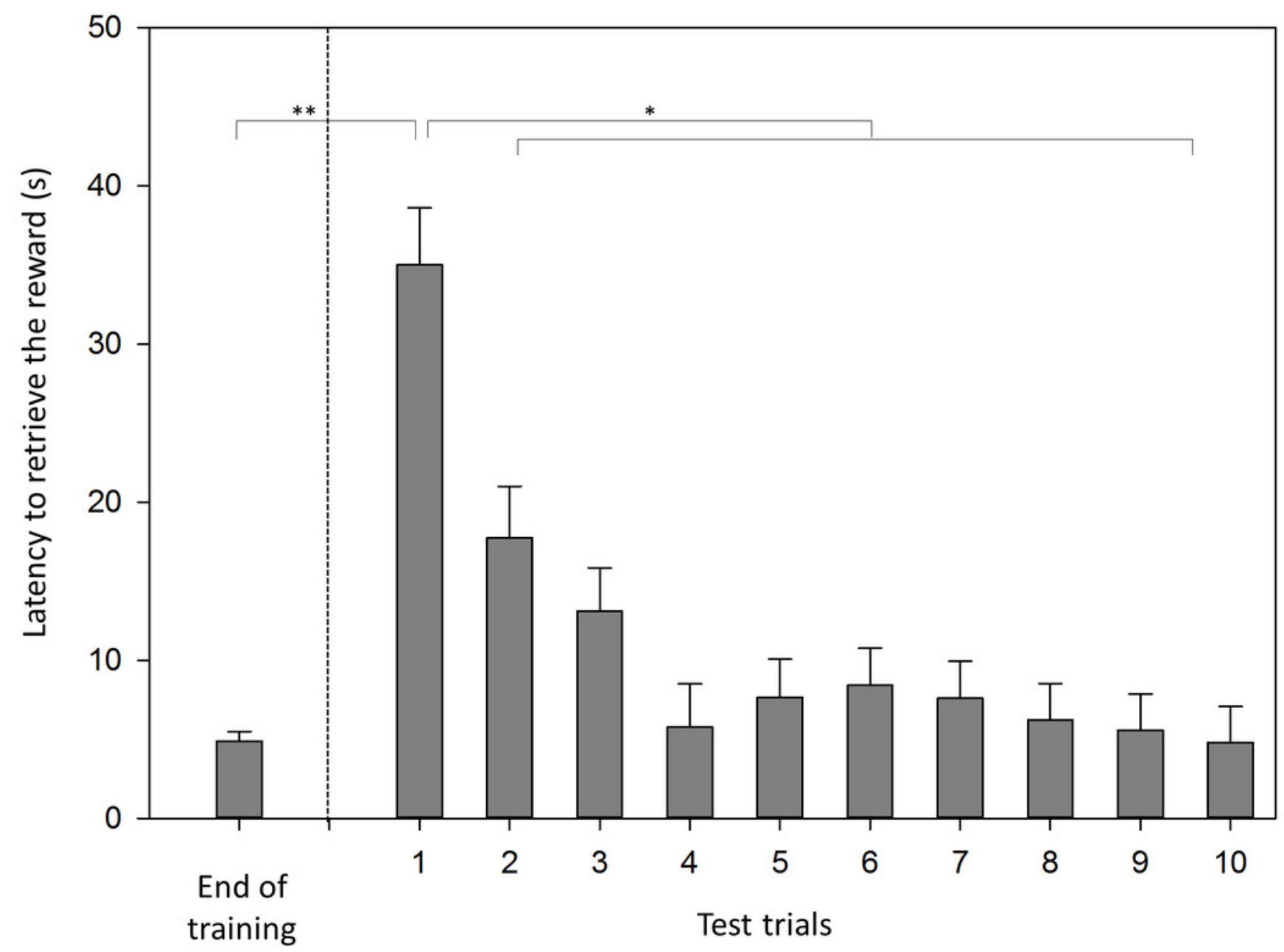




\section{Figure 5}

Relationship between brain size and motor self-regulation

Relationship between absolute brain size and performance in the cylinder task for 26 mammal species from 4 orders. The trend line is based on a regression across all species. The two domestic animals species are marked in red. (1, Mongolian gerbil; 2 , marmoset; 3 , fox squirrel; 4, golden-headed lion tamarin; 5 , mongoose lemur; 6 , black lemur; 7, ring-tailed lemur; 8, squirrel monkey; 9, brown lemur; 10, Coquerel's sifaka; 11, red-bellied lemur; 12, ruffed lemur; 13 , aye aye; 14, capuchin monkey; 15 , coyote; 16, domestic dog; 17, rhesus macaque; 18, golden snub-nosed monkey; 19, grey wolf; 20, domesticated goat; 21 , hamadryus baboon; 22, olive baboon; 23 , bonobo; 24 , chimpanzee; 25 , orangutan; 26 , gorilla [MacLean et al. 2014; Ballarin et al. 2016]).

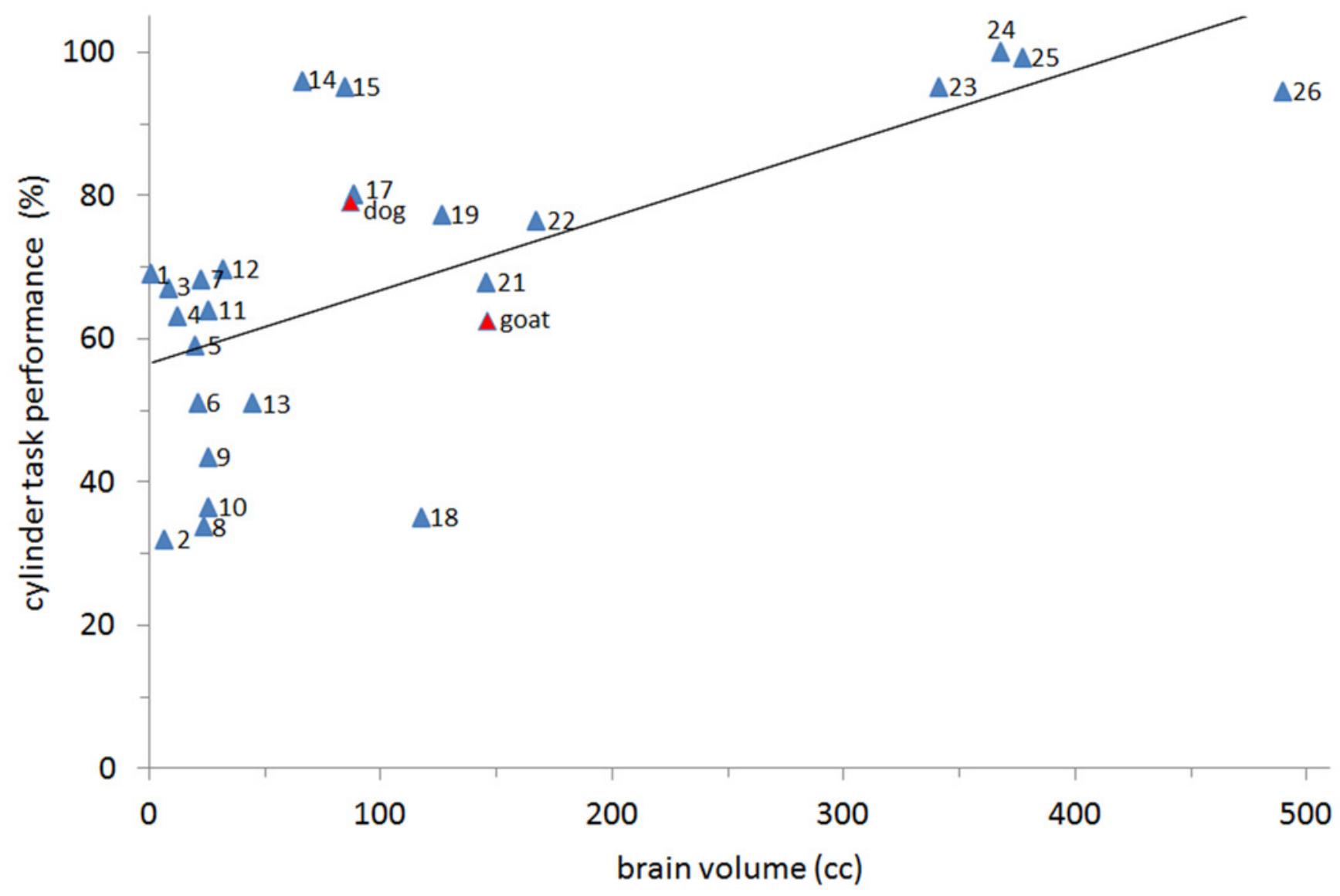

